\title{
Is Cartridge Based Nucleic Acid Amplification Test (CBNAAT) Better Than Conventional Tests in Diagnosing Childhood Tuberculosis? Evidence from a Tertiary Care Hospital in Eastern India
}

\author{
Sandip Sen ${ }^{1}$, Anjan Kumar Das ${ }^{2 *}$, Debanjan Sinha ${ }^{3}$ \\ ${ }^{1}$ Associate Professor, ${ }^{2}$ Assistant Professor, ${ }^{3}$ Post Graduate Trainee, Dept. of Paediatrics, Dr. B. C. Roy Post Graduate Institute of Paediatric Sciences, \\ 111 Narkeldanga Main Road, Kolkata 700054, West Bengal, India
}

DOI: $10.36348 /$ sjmps.2019.v05i11.013 $\quad$ | Received: 06.11.2019| Accepted: 22.11 .2019 | Published: 30.11 .2019

*Corresponding author: Anjan Kumar Das

\section{Abstract}

Introduction: Tuberculosis is one of the major causes of mortality and morbidity in childhood. It is challenging to diagnose in childhood population due to difficulty in collection of sample and paucibacillary nature. Despite of considering mycobacterium culture as gold-standard test, it is time consuming (4-8 weeks) while traditional ZN stain is poorly sensitive or specific. In 2013, WHO endorsed Cartridge Based Nucleic Acid Amplification Test (CBNAAT) for diagnosis of TB. Still, there is lack of adequate research on TB detection efficacy of CBNAAT over ZN stain or culture. Aims \& Objective: Comparison of CBNAAT over ZN stain \& mycobacterium culture, in terms of Sensitivity, Specificity, Positive Predictive Value, Negative predictive value. Materials \& Methods: Total of 565 patients admitted to Paediatric Medicine department over 2 years from July 2017 to July 2019 with clinical signs (radiological) and symptoms of local \& disseminated tuberculosis and history of close contact were selected and grouped under pulmonary and extrapulmonary categories. Each sample was analyzed by CBNAAT, ZN smear and culture. Results: In the pulmonary group, CBNAAT detected 43 out of 185 sputum samples (23.2\%), 110 out of 308 gastric lavage samples (35.7\%), 0 out of 4 BAL samples. Besides, in extrapulmonary group- 6 out of 41 CSF samples, 3 out of 15 pleural fluid, 0 out of 7 ascitic fluid and 3 out of 5 LN biopsy samples were detected by CBNAAT. Sensitivity, specificity, positive predictive value, negative predictive value of CBNAAT in reference to $\mathrm{ZN}$ stain was $100 \%, 92.1 \%, 70.6 \%$ and $100 \%$ while in reference to culture was $93.2 \%, 99.1 \%, 96.2 \%$ and $97.2 \%$ respectively. Conclusion: CBNAAT assay is highly sensitive and specific to detect tuberculosis rapidly comparing to the traditional tests with rifampicin resistance associated with mutation of rpoB gene in a single test. It also helps in avoiding injudicious use of anti-tubercular drugs.

Keywords: CBNAAT, MTB, ZN smear, Culture.

Copyright @ 2019: This is an open-access article distributed under the terms of the Creative Commons Attribution license which permits unrestricted use, distribution, and reproduction in any medium for non-commercial use (NonCommercial, or CC-BY-NC) provided the original author and source are credited.

\section{INTRODUCTION}

Tuberculosis $(\mathrm{TB})$ is the $10^{\text {th }}$ leading cause of death worldwide, and since 2007 it is the single largest cause of death from a single infectious agent, more than HIV/AIDS and malaria. Most of these deaths could be prevented with early diagnosis and appropriate treatment. For example, among people whose TB was detected, reported and treated in 2017, the treatment success rate was $85 \%$ globally; and in high income countries with UHC (Universal Health Care), the proportion of people who die from TB can be under $5 \%$ [1].

The incidence of $\mathrm{TB}$ in the paediatric age group (0-14 years) is 6\% according to Global Tuberculosis Report 2019 and India TB Report 2019 although regional differences do exist as the state of study (West Bengal) has reported $4 \%$ incidence. The hospital associated DTC (District TB Centre) has reported an incidence $9.6 \%$ and $8 \%$ in the year 2019 due to its urban location and referral bias. Delhi and Chandigarh has reported a high incidence for the same reason $[1,2]$.

Pulmonary TB is most common in pediatric age group, while extra-pulmonary cases form a larger proportion comparing the adults. It is challenging to diagnose pediatric TB with microbiological tests due to its paucibacillary nature as well as difficulty to obtain good quality of specimen [3, 4]. In adult pulmonary TB, it is easily picked up by the sputum (specimen) smear examination, while sputum is generally swallowed up by the children. The gold standard test, that is, culture (solid or liquid) from the specimen usually takes 4-8 
weeks to give the appropriate results and also require expensive and sophisticated laboratory facilities which is often challenging in resource poor settings [4]. There are some other tests like antigen-antibody ELISA tests, Interferon gamma release assay (IGRA) tests, but their specificity and sensitivity to detect pediatric $\mathrm{TB}$ is highly questionable. WHO, in a policy statement, has strongly recommended that serological tests not to be used in diagnosis of TB [5].

Since 2013, Cartridge Based Nucleic Acid Amplification Test (CBNAAT) has been introduced for diagnostic purpose to detect pediatric tuberculosis (pulmonary and extrapulmonary). It has been endorsed by WHO and with an advantage of quick turnaround time also simultaneously detects $\mathrm{TB}$ and rifampicin resistance thus providing a promising solution to achieve early TB detection and hence better care and control of TB [6]. However, the sensitivity and specificity of the new test has not been adequately researched in comparison to simple $\mathrm{ZN}$ stain or gold standard culture test.

So, we have aimed to conduct this study to compare the utility and importance of CBNAAT with some traditional tests in terms of sensitivity, specificity, positive as well as negative predictive values to pick up the pulmonary and extrapulmonary focus of tuberculosis rapidly and appropriately.

\section{MATERIALS AND METHODS}

The study was conducted at Pediatric Medicine department of Dr. BC Roy Post Graduate Institute of Pediatric Sciences, Kolkata from July 2017 to July 2019, after taking the necessary Institutional Ethics Committee approval. Total 565 patients showing symptoms and signs of suspected localized and/ or disseminated tuberculosis or history of contact with diagnosed tuberculosis were included in the study excluding the patients whose parents did not give their consent to participate.

\section{STUDY TOOLS}

Necessary history was taken and clinical examination done. All patients were evaluated for pulmonary symptoms like fever, cough \& cold, hemoptysis as well as extrapulmonary symptoms like chest pain, bone pain, headache, seizures etc.

Case Definition: TB suspect-children with persistent fever and/or cough for more than 2 weeks, loss of weight/no weight gain (History of unexplained weight loss or no weight gain in past 3 months; loss of weight is defined as loss of more than $5 \%$ body weight as compared to highest weight recorded in last 3 months) and/or history of contact with infectious TB cases (In a symptomatic child, contact with a person with any form of active TB within last 2 years may be significant) [7].
Presumptive extrapulmonary TB- presence of organ specific symptoms and signs like swelling of lymph node, pain and swelling in joints, neck stiffness, disorientation, etc and/or constitutional symptoms like significant weight loss, persistent fever for more than 2 weeks, night sweats.

Laboratory Investigations such as complete blood count (CBC), erythrocyte sedimentation rate (ESR), Mantoux test, Zeihl Neelsen (ZN) smear, fine needle aspiration cytology (FNAC), histopathology of samples and culture.

Imaging studies including chest skiagram, ultrasonography, computed tomography (CT) scan, magnetic resonance imaging (MRI) scan.

CBNAAT of sputum/induced sputum, gastric aspirate/lavage, cerebrospinal fluid, pleural fluid, ascitic fluid, bronchoalveolar lavage (BAL), FNAC material.

\section{STUDY TECHNIQUE}

Patients were selected as per the inclusion criteria and recruited in the study. Detailed history taking, physical examination and relevant laboratory investigations were done. A pre designed semi structured proforma was used to obtain data based on socio-economic profile, clinical profile and investigations after explaining the purpose of the study and obtaining informed consent from the parent/guardian of the child in writing.

All the samples were collected in well labelled falcon tubes. In pulmonary cases two sputum/induced sputum (Sputum induction was done in a wellventilated room with an ultrasonic nebuliser and nebulisation done with $10-20 \mathrm{~mL}$ of $3 \%$ hypertonic saline until patient coughed up at least $2 \mathrm{~mL}$ of sputum or a maximum of 15 minutes). Samples were collected: one early morning and other supervised spot specimen. Smears of both the sputum samples were made, stained by $\mathrm{ZN}$ procedure, examined under light microscope. Thereafter, if the samples were positive for acid fast bacilli (AFB), then the positive sample otherwise good quality or early morning sample was used for CBNAAT. So, only one sample was further processed for CBNAAT. In infants and small children gastric lavage was collected in place of sputum. Where invasive techniques like BAL were required, only one sample was collected. Bronchoscopy was done with Pentax fibre optic bronchoscope (FOB) under local anaesthesia with $4 \%$ lignocaine jelly and mouth spray. Bronchial washing and lavage was performed by instilling $20 \mathrm{~mL}$ aliquots of normal saline at room temperature up to $100-120 \mathrm{~mL}$ and collected into a sterile suction trap by aspiration. In extrapulmonary cases the samples collected were CSF by lumbar puncture (LP), lymph node fluid by FNAC, pleural fluid by pleural tap and ascitic fluid by ascitic tap. 


\section{CBNAAT}

CBNAAT samples were sent to District Tuberculosis Centre (DTC) (Tangra Chest Clinic) at 15/1 Baishali, Gobindo Khatick Road, Kolkata 46.CBNAAT was performed according to the manufacturer's instructions (CEPHEID, Sunnyvale, CA, USA). Our machine contains 4 cartridges so 4 samples were processed for each run. According to standard operating procedure the sampling reagent (containing $\mathrm{NaOH}$ and isopropanol) was added at 2:1 ratio to the sample and kept for 15 minutes at room temperature with intermittent shaking. $3 \mathrm{ml}$ of this treated sample was transferred to the cartridge and the cartridge was inserted in the module of CBNAAT machine.

An automatic process completed the remaining assay steps and the results were displayed on the monitor attached to Gene Xpert after $1 \mathrm{hr}$ and 50 minutes.

\section{AFB Smear}

A minimum of 1 slide positive even for single $\mathrm{AFB} / 100$ fields was taken as positive for Mycobacterium tuberculosis and a minimum of two samples negative for AFB evaluated for 100 fields were declared as negative.

\section{Culture}

The concentrated sample obtained after decontamination was inoculated for culture in
BACTEC mycobacterium growth indicator tube (MGIT) for liquid culture. Culture was considered as gold standard for analysis of results. When the tubes were flagged positive by the system, $\mathrm{ZN}$ staining and culture on 5\% sheep blood agar were performed from the tube directly to see any contamination as per the manufacturer's instructions. All tubes were checked for positivity till 42 days. Mycobacteria other than tuberculosis (MOTT) and Mycobacterium tuberculosis testing from positive culture tubes were done by rapid immunochromatography test kit using MPT 64 antigen according to the manufacturer's instructions.

\section{Data Analysis}

All recorded data were analyzed using standard statistical methods (SPSS software). Sensitivity, specificity, positive predictive value and negative predictive value of CBNAAT were calculated. Values of $\mathrm{P}<0.05$ were considered as statistically significant.

\section{RESULTS}

Total 565 patients were examined for this study, youngest patient being 5 months and oldest patient were 11 years 3 months. Table- 1 shows that the majority of the patients were males $66.7 \%$ and belonged to the age group $<5$ years $(45.1 \%)$. Only $18 \%$ showed history of TB contact while $16 \%$ patients showed Mantoux positivity.

Table-1: Demographic Profile of the Cases

\begin{tabular}{|l|l|l|}
\hline PARAMETERS & NO OF CASES & PERCENTAGE (\%) \\
\hline GENDER & & \\
\hline Male & 377 & 66.7 \\
\hline Female & 188 & 33.3 \\
\hline AGE GROUP & & \\
\hline$<5$ years & 255 & 45.1 \\
\hline 5-9 years & 205 & 36.2 \\
\hline 9-12 years & 105 & 18.6 \\
\hline $\begin{array}{l}\text { HISTORY OF CONTACT WITH } \\
\text { TB PATIENTS }\end{array}$ & & \\
\hline Yes & 102 & 18.1 \\
\hline No & 463 & 81.9 \\
\hline MANTOUX TEST & & \\
\hline Positive & 91 & 16.1 \\
\hline Negative & 474 & 83.9 \\
\hline
\end{tabular}

Table-2: Type and Number of Samples Used

\begin{tabular}{|l|l|l|l|}
\hline & Type of sample & Total samples & MTB detected by CBNAAT \\
\hline \multirow{4}{*}{$\begin{array}{l}\text { PULMONARY }(\mathrm{n}=497) \\
\end{array}$} & Sputum/ Induced sputum & 185 & $43(23.2 \%)$ \\
\cline { 2 - 4 } & Gastric lavage/ aspirate & 308 & $110(35.7 \%)$ \\
\cline { 2 - 4 } & Broncho-alveolar lavage & 4 & $0(0 \%)$ \\
\cline { 2 - 4 } & TOTAL & 497 & $153(30.8 \%)$ \\
\hline \multirow{3}{*}{$\begin{array}{c}\text { EXTRA PULMONARY } \\
(\mathrm{n}=68)\end{array}$} & CSF & 41 & $6(14.6 \%)$ \\
\cline { 2 - 4 } & Pleural fluid & 15 & $3(20 \%)$ \\
\cline { 2 - 4 } & Ascitic fluid & 7 & $0(0 \%)$ \\
\cline { 2 - 4 } & FNAC material from lymph node & 5 & $3(60 \%)$ \\
\cline { 2 - 4 } & TOTAL & 68 & $12(17.6 \%)$ \\
\hline
\end{tabular}

CBNAAT detected 132 out of 140 culture positive cases $(94.3 \%)$ and in 6 of the culture negative cases $(1.85 \%)$. 
Table-3: Performance of CBNAAT versus CULTURE

\begin{tabular}{|l|l|l|l|}
\hline & Culture positive & Culture negative & Total \\
\hline CBNAAT positive & 132 & 6 & 165 \\
\hline CBNAAT negative & 8 & 319 & 400 \\
\hline TOTAL & 140 & 325 & 565 \\
\hline
\end{tabular}
$(13.3 \%)$.

CBNAAT detected 105 out of $116 \mathrm{ZN}$ stain positive cases $(90.5 \%)$ and 60 out of $449 \mathrm{ZN}$ stain negative cases

Table-4: Performance of CBNAAT versus ZN Smear

\begin{tabular}{|l|l|l|l|}
\hline & ZN stain positive & ZN stain negative & Total \\
\hline CBNAAT positive & 105 & 60 & 165 \\
\hline CBNAAT negative & 11 & 389 & 400 \\
\hline TOTAL & 116 & 449 & 565 \\
\hline
\end{tabular}

Sensitivity, specificity, positive predictive value and negative predictive value of CBNAAT in reference to $\mathrm{ZN}$ stain and Culture.

Table-5

\begin{tabular}{|l|l|l|l|l|}
\hline & Sensitivity & Specifity & Positive predictive value & Negative predictive value \\
\hline CBNAAT vs Culture & $94.3 \%$ & $89.8 \%$ & $80 \%$ & $97.3 \%$ \\
\hline CBNAAT vs ZN stain & $90.5 \%$ & $86.6 \%$ & $63.6 \%$ & $97.3 \%$ \\
\hline
\end{tabular}

\section{DISCUSSION}

The WHO policy update in 2017 for Automated real-time nucleic acid amplification technology for rapid and simultaneous detection of tuberculosis and rifampicin resistance: Xpert MTB/RIF assay for the diagnosis of pulmonary and extrapulmonary $\mathrm{TB}$ in adults and children has recommended that in children, Xpert MTB/RIF may be used rather than conventional microscopy and culture as the initial diagnostic test in all children suspected of having TB (conditional recommendation acknowledging resource implications, very low-quality evidence). Also Xpert MTB/RIF should be used rather than conventional microscopy, culture and DST as the initial diagnostic test in children suspected of having MDR-TB or HIV-associated TB (strong recommendation, very low-quality evidence) [6]. Hence we can conclude that high -quality evidence is lacking in children as compared to adults. This study attempts to give evidence from Eastern India but lacks robust data on MDR TB and Rifampicin resistance.

There are only a few studies on CBNAAT assay to detect tuberculosis in India. CBNAAT was developed by the Foundation for Innovative New Diagnostics (FIND), with the Cepheid Corporation and the University of Medicine and Dentistry of New Jersey. The basic principle is to detect targeted DNA sequences of Mycobacterium tuberculosis genome by real time PCR method, as well as rifampicin resistance and results can be obtained within 2 hours.

We have included 565 patients in this group with the above mentioned inclusion \& exclusion criteria. The youngest one was 5 months while the oldest one was 11 yr 3 months with male: female ratio $2: 1$. Majority of the children were $<5$ years age group
(45.1\%). Mantoux test was positive among $16 \%$ patients while $18 \%$ showed no history of contact. There were 497 pulmonary and 68 extra pulmonary cases. Among the pulmonary cases, CBNAAT detected 43 out of 185 sputum or induced sputum sample (23.2\%), 110 out of 308 gastric lavage or aspirate sample (35.7\%), 0 out of 4 samples of broncho-alveolar lavage, in total 153 pulmonary samples were detected for MTB by CBNAAT out of 497 samples $(30.8 \%)$. Besides, in extra pulmonary group, 12 out of 68 samples were detected for tuberculosis, which includes 6 out of 41 CSF samples (14.6\%), 3 out of 15 pleural fluid samples (20\%), 0 out of 7 ascitic fluid sample, 3 out of 5 FNAC material from lymph node $(60 \%)$. Culture detected 140 out of 565 cases $(24.8 \%)$ and ZN stain detected 116 out of 565 cases $(20.5 \%)$.

CBNAAT detected 132 out of 140 culture positive patients $(94.2 \%), 6$ out of 325 culture negative patients $(0.02 \%)$. So, 6 were false positive as CBNAAT positive despite of gold standard culture report being negative. This was due to incomplete anti tubercular treatment taken by those 6 cases. CBNAAT can detect nucleic acid even in dead bacilli too. ZN stain was positive among 105 out of 165 CBNAAT positive case $(63.6 \%)$ s as well as 11 out of 400 CBNAAT negative cases $(0.03 \%)$. Those 11 cases may be due to very low bacterial load, too low to detect the DNA from MTB complex, to be detected by CBNAAT.

Sensitivity, specificity, positive and negative predictive values of CBNAAT in reference to gold standard culture test were $94.3 \%, 89.8 \%, 80 \%$ and $97.3 \%$ respectively. In the study of Sowjanya (Andhra Pradesh, India) (June 2012- December 2013), CBNAAT detected MTB in 144 out of 205 pulmonary specimens $(70.24 \%$ detection rate) 
whereas sputum for AFB was able to detect only 108 cases $(52.68 \%$ detection rate). CBNAAT detected 108 out of the 109 sputum smear positive cases, and 36 out of the 96 sputum smear negative cases [9]. Our study showed that CBNAAT detected 153 of 497 pulmonary cases $(30.8 \%)$ and 12 of 68 extrapulmonary cases $(17.6 \%)$. The difference of result may be due to the Andhra study being an adult study and our study being a paediatric study where the pauci bacillary cases predominate and the bacterial load is less. It was found in a study by Singh in Delhi that of 50 individuals aged $0-14$ years with suspected PTB, 23 (46\%) were diagnosed with PTB based on the RNTCP algorithm [10]. Sixteen children from the PTB group (69.5\%) were Xpert-positive. None in the 'not PTB' group were Xpert-positive. With culture as gold standard, Xpert sensitivity and specificity were respectively $91.6 \%$ (95\% CI 59.7-99.5) and $86.8 \%$ (95\% CI 71.1-95.05). This compares favourably with our data showing a Xpert sensitivity of $94.3 \%$ and $89.8 \%$ specificity as compared to culture methods.

Sensitivity, specificity, positive and negative predictive values of CBNAAT in reference to $\mathrm{ZN}$ smear were $90.5 \%, 86.6 \%, 63.6 \%$ and $97.3 \%$ respectively. It is evident that CBNAAT is superior to $\mathrm{ZN}$ smear for diagnosing paediatric TB. CBNAAT detected 60 out of 449 smear negative cases (13.4\%). CBNAAT also detected 10 cases of rifampicin resistance among the 165 cases detected by CBNAAT (6.1\%). First National Drug Resistance Survey results showed the rates of MDR are $6.19 \%$ overall and among new TB patients to be $2.84 \%$ and that in previously treated to be $11.60 \%$ [11]. It is difficult to draw conclusions as good quality paediatric MDR TB data are lacking and the above study gives adult data and was powered for national data as compared to state wise data.

In a 4 city urban Indian paediatric study involving 8370 presumptive paediatric TB cases of which were 517 Xpert/CBNAAT positive cases by Raizada et al., [12], more than 2 fold higher TB case detection was observed on Xpert as compared with smear microscopy irrespective of type of specimen. The increase in TB detection rate was highest while testing CSF, fine needle aspiration cytopathology samples, BAL and Pus specimen with Xpert as compared to smear microscopy. It was observed that none of the 52 ascetic fluid specimen tested had a positive test result either on Xpert or smear microscopy. Also, there was no significant gain in TB detection (OR 1.2 (95\% CI 0.3-4.0) on Xpert over smear microscopy in case of pleural fluid. This is in consonance with WHO guidelines issued which prefers a pleural biopsy over pleural fluid sample [6]. Our study also showed that pleural fluid was positive for MTB by CBNAAT in 3 of 15 cases (20\%) only and none for 7 ascitic fluid samples $(0 \%)$.
The meta-analysis by Maynard-Smith involving 6026 extrapulmonary TB samples from 27 studies included in this review showed that median sensitivity and specificity of Xpert assay with a culture based reference standard were 0.83 (IQR, $0.68-0.94)$ and 0.98 (IQR, 0.89-1.00) respectively [13]. An important unresolved issue regarding the use of Xpert assay to test non-respiratory samples is the differing processing requirements for each sample type. Technical studies on optimum processing methods would enable the highest rates in sensitivity to be achieved, as well as lowering indeterminate rates. Denkinger (USA) performed a systematic review and meta- analysis to assess the accuracy of Xpert for the detection of extrapulmonary TB [14]. They determined the accuracy of Xpert compared with culture and a composite reference standard (CRS). They identified 18 studies involving 4461 samples. In lymph node tissues or aspirates, Xpert pooled sensitivity was $83.1 \%$ (95\% CI 71.4-90.7\%) versus culture and $81.2 \%$ (95\% CI $72.4-87.7 \%$ ) versus CRS. In cerebrospinal fluid, Xpert pooled sensitivity was $80.5 \%$ (95\% CI 59.0-92.2\%) against culture and62.8\% (95\% CI 47.7-75.8\%) against CRS. In pleural fluid, pooled sensitivity was $46.4 \%$ (95\% CI26.3-67.8\%) against culture and $21.4 \%$ (95\% CI 8.8-33.9\%) against CRS. Xpert pooled specificity was consistently $>98.7 \%$ against CRS across different sample types. Based on this systematic review, the World Health Organization now recommends Xpert over conventional tests for diagnosis of TB in lymph nodes and other tissues, and as the preferred initial test for diagnosis of TB meningitis.

Thus, this study adds data from TB endemic third world country. CBNAAT can help in rapid diagnosis of pediatric tuberculosis with high sensitivity, specificity, positive and negative predictive value. It also detects rifampicin resistance allowing for appropriate treatment to be started early. This is also cost saving by detecting true TB negative patients by avoiding unnecessary treatment.

However, CBNAAT assay has a lot of disadvantages [6]:

1. Not suitable to assess the response to treatment like conventional microscopy and culture.

2. Several technical problems like requirement of stable electricity, limited temperature change ( 2 to $28 \mathrm{deg}$ C), availability of maintenance, annual calibration, limited throughput at 16-20 samples per day in busy centres, cartridge shelf life of 12 months.

3. It cannot detect emerging rifampicin resistance during treatment as well as isoniazid monoresistance.

4. Unable to differentiate between XDR-TB from MDR-TB as it detects only rifampicin 
resistance. Also rifampicin resistance can only be detected if rpoB allele is present in at least $65 \%$ of DNA present in sample.

5. It cannot be used in blood, stool and urine samples and gives a poor yield in pleural and ascetic fluid specimens.

Multicentre study with larger sample can help in further evaluation of result and statistical analysis with positive and negative likelihood ratio can help in further evaluation of the results statistically.

\section{CONCLUSION}

CBNAAT assay has revolutionized TB diagnosis and treatment by allowing identification of MTB with high specificity, sensitivity, positive predictive value and negative predictive value along with rifampicin resistance. It is also cost effective as compared to culture methods and gives us results in 2 hours. Appropriate treatment and early control measures can be initiated for MDR-TB cases, thereby ultimately reducing incidence and avoiding injudicious use of anti-tuberculosis drugs. WHO and RNTCP guidelines recommend its use in diagnosing pulmonary $\mathrm{TB}$, extra pulmonary $\mathrm{TB}$, paediatric $\mathrm{TB}$ as well as rifampicin resistance and MDR-TB in high risk populations like People living with HIV infection (PLHIV).Further research is required to overcome the disadvantages as already mentioned and to reduce its cost for which measures are already underway.

\section{Funding: None}

Conflicts of interest: The authors report no conflicts of interest. The authors alone are responsible for the content and writing of this article. All authors approved the final version of manuscript and agree to be accountable for authenticity and integrity of the work.

\section{REFERENCES}

1. Global tuberculosis report 2019. Geneva: World Health Organization; 2019. https://apps.who.int/iris/bitstream/handle/10665/3293 68/9789241565714-eng.pdf?ua=1 accessed on 18 Nov 2019.

2. India TB Report 2019. New Delhi, Central Tuberculosis Division, Dept. of Health \& Family Welfare:Govt. of India; 2019. https://tbcindia.gov.in/index1.php?lang=1\&level=2\& sublinkid $=5358 \&$ lid $=3450$ accessed on 18 Nov 2019 .

3. Raj, A., Singh, N., \& Mehta, P. K. (2012). GeneXpert MTB/RIF assay: a new hope for extrapulmonary tuberculosis. IOSR J Pharm,2(1), 83-89.

4. Haldar, S., Bose, M., Chakrabarti, P., Daginawala, H. F., Harinath, B. C., Kashyap, R. S., ... \& Srivastava, R. (2011). Improved laboratory diagnosis of tuberculosis-the

Indian

experience. Tuberculosis, 91(5), 414-426.

5. WHO Tuberculosis Serodiagnostic Tests Policy Statement 2011. Geneva: World Health Organization; 2011. https://www.who.int/tb/areas-ofwork/laboratory/serology.pdf accessed on 18 Nov 2019.

6. WHO Xpert MTB/RIF Assay for the diagnosis of pulmonary and extrapulmonary $\mathrm{TB}$ in adults and children 2017. Geneva: World Health Organization; 2017. https://www.who.int/tb/publications/xpert-mtbrif-assay-diagnosis-policy-update/en/ accessed on 18 Nov 2019.

7. Guidelines on Paediatric Tuberculosis. India TB Report 2019. New Delhi, Central Tuberculosis Division, Dept. of Health \& Family Welfare:Govt. of India; 2019. https://tbcindia.gov.in/index1.php?sublinkid=4200\&1 evel=2\&lid $=2848 \&$ lang $=1$ accessed on 18 Nov 2019 .

8. Barnard, D. A., Irusen, E. M., Bruwer, J. W. Plekker, D., Whitelaw, A. C., Deetlefs, J. D., \& Koegelenberg, C. F. (2015). The utility of Xpert MTB/RIF performed on bronchial washings obtained in patients with suspected pulmonary tuberculosis in a high prevalence setting. BMC pulmonary medicine, 15(1), 103.

9. Sowjanya, D. S., Behera, G., Reddy, V. R., \& Praveen, J. V. (2014). CBNAAT: a novel diagnostic tool for rapid and specific detection of mycobacterium tuberculosis in pulmonary samples. International Journal of Health Research in Modern Integrated Medical Sciences, 1(1):28-31.

10. Singh, M., Sethi, G. R., Mantan, M., Khanna, A., \& Hanif, M. (2016). Xpert ${ }^{\circledR}$ MTB/RIF assay for the diagnosis of pulmonary tuberculosis in children. The International Journal of Tuberculosis and Lung Disease, 20(6), 839-843.

11. Ministry of Health and Family Welfare GoI. (2018). Report of the first national anti-tuberculosis drug resistance survey: India 2014-16. https://tbcindia.gov.in/showfile.php?lid=3315 (accessed on 18 Nov 2019.

12. Raizada, N., Sachdeva, K. S., Swaminathan, S., Kulsange, S., Khaparde, S. D., Nair, S. A., ... \& Umadevi, K. R. (2015). Piloting upfront Xpert MTB/RIF testing on various specimens under programmatic conditions for diagnosis of TB \& DR$\mathrm{TB}$ in paediatric population. PLoS One, 10(10), e0140375.

13. Maynard-Smith, L., Larke, N., Peters, J. A., \& Lawn, S. D. (2014). Diagnostic accuracy of the Xpert MTB/RIF assay for extrapulmonary and pulmonary tuberculosis when testing non-respiratory samples: a systematic review. BMC infectious diseases, 14(1), 709.

14. Denkinger, C. M., Schumacher, S. G., Boehme, C. C., Dendukuri, N., Pai, M., \& Steingart, K. R. (2014). Xpert MTB/RIF assay for the diagnosis of extrapulmonary tuberculosis: a systematic review and meta-analysis. European Respiratory Journal, 44(2), 435-446. 